\title{
ISABELLA BIRD: UNA MIRADA FEMENINA EN LA CUMBRE ${ }^{1}$
}

\author{
Iratxe Ruiz-de-Alegría Puig ${ }^{2}$
}

\section{Isabella Bird: una mirada femenina en la cumbre}

Resumen: El objetivo del artículo consiste en explorar las maneras en que la viajera y escritora británica Isabella Bird entiende el placer visual ante el paisaje de montaña, ofreciendo una perspectiva anticipada y atrevida para su época que da a conocer una estética sensual desde la experiencia femenina, blanca, y heterosexual. A partir de una selección de cartas de la autora a su hermana, posteriormente publicadas, se ha enfrentado la "mirada femenina" con la "masculina". Tras su análisis, el discurso de Bird resulta tener un trasfondo mucho más significativo en cuanto a la subversión de la imperante mirada patriarcal sobre la montaña en Occidente.

Palabras clave: Mirada femenina, montaña metafórica, cuerpo masculino, paisaje subversivo.

\section{Isabella Bird: a Female Gaze at the Apex}

Abstract: The present article aims at exploring the ways in which the British traveller and writer Isabella Bird understands visual pleasure before a mountainous landscape. Bird's reading seems to be pioneering and courageous for a Victorian Lady, not least because she offers a sensual aesthetic from a female, white, and heterosexual perspective. From a selection of missives Bird sent to her sister, subsequently published, I opposed the "female gaze" to the "male gaze". The results obtained show that the traveller's discourse turns out to be of remarkable significance regarding the subversion of the patriarchal leading gaze on the mountain in the western culture.

Key words: Female gaze, metaphoric mountain, male body, subversive landscape.

\section{Introducción}

What is the use of a house if you haven't got a tolerable planet to put it on? (¿De qué sirve una casa si no se cuenta con un planeta aceptable en el que colocarla?) ${ }^{3}$

Henry David Thoreau (Letters to a Spiritual Seeker, 1860)

\footnotetext{
${ }^{1}$ Fecha de recepción: 15/11/2019.

Fecha de aceptación: 03/05/2020.

2 Profesora en la Escuela Oficial de Idiomas de Bilbao, País Vasco, España; $\bowtie$ iruiz.de.alegria@irakasle.eus.

${ }^{3}$ Traducción propia.
} 
Fue a partir de la filosofía Kantiana que la categoría del espacio adquirió mayor relevancia, no solo en el ámbito de la literatura sino en el del arte también, puesto que tanto los escritores como los artistas proyectan sus preocupaciones y expresan sus ideas a través de las dimensiones u objetos del espacio (Garrido Domínguez 2007: 207). Lo cierto es que, inducido por el deseo de conocer, medir, controlar y adueñarse del espacio, el hombre en masculino se apropió indebidamente de todos los elementos que en él habitaban elaborando un entramado complejo de simbologías e identificaciones conforme a sus gustos (blanco, masculino, heterosexual) que han perdurado a lo largo de la historia (Rose 1993; Griffin 2015). En este artículo presento un ejemplo de espacio en femenino (mujer, blanca, heterosexual), puesto que como indica la filósofa $y$ activista feminista Victoria Sendón: “Occidente únicamente conoce la historia contada por el Padre" (citado en Alario 2008: 64). Por tanto, propongo centrarnos esta vez en la historia contada por la Madre. Para ello, ofrezco un primer apartado en el que explico la construcción de una iconografía, producto exclusivo de la mirada blanca, masculina, heterosexual mencionada anteriormente. Consagro el segundo apartado al espacio narrativo (Álvarez 2002) presentado en la novela epistolar de Isabella Bird: La vida de una mujer en Las montañas Rocosa $(1879)^{4}$. Del examen de dicho espacio, siguiendo el interesante modelo propuesto por la experta en Igualdad, Eva $\mathrm{M}^{\mathrm{a}}$ Antón Fernández (2017), deduzco que, en la literatura en inglés del siglo XIX, ya existía un interés, una preocupación, y una clara militancia en favor de la Naturaleza. Así mismo, infiero el nacimiento de una particular posición para su observación (mujer, blanca, heterosexual), alternativa a la mirada tradicional masculina omnisciente en el mundo occidental, que pone en cuestión la manida identificación del cuerpo femenino con la Naturaleza, y el encasillamiento en su rol reproductivo (Carretero 2010; Puleo 2013; Antón 2015).

\footnotetext{
${ }^{4}$ Todas las citas referidas a la obra de Isabella Bird han sido extraídas de su correspondiente traducción al castellano: Una mujer en las montañas Rocosas, Carlos Ezquerra (trad.), 2014.
} 


\section{La apropiación del espacio: el nacimiento del paisaje}

Seeing comes before words. (La vista precede a las palabras) ${ }^{5}$ John Berger (Ways of Seeing, 1972)

De acuerdo con el geógrafo británico Dennis E. Cosgrove (1985), el paisaje es un término acuñado durante el Renacimiento en su obsesión por controlar y dominar el espacio (46). La burguesía enriquecida con el florecimiento del comercio buscaba incesantemente cartografiar y controlar sus propiedades recientemente adquiridas. Por tanto, no es fruto del azar que los comerciantes de los siglos XV y XVI, erigidos en la nueva clase dominante, comisionaran retratos al óleo para alardear de sus respectivas propiedades (46-47). A ese respecto, Cosgrove (1983) apunta que "la ideología se apropia y reproduce el espacio para legitimar y mantener a la clase dominante" ${ }^{\circ}(10)$. Esa pintura al óleo se encuentra, para el gran experto en veredas y naturaleza Robert Macfarlane (2016), indisociablemente unida al paisaje puesto que se elabora con minerales extraídos de la propia tierra. Macfarlane añade que "se ha forzado el empleo de la tierra para obligarla a expresarse por sí misma"7 (2016: 793-794). Por otro lado, tomando como modelo algunos de los ejemplos más debatidos por los geógrafos, la británica Gillian Rose (1993) analiza, entre otras, la pintura: Las mujeres tahitianas (1891), de Gauguin. En primer lugar, Rose denuncia la mirada que observa, que no es otra que la mirada masculina heterosexual, única responsable, en su opinión, del rol puramente decorativo de aquellas, en perfecto estatismo, ofreciendo sus pechos

\footnotetext{
${ }^{5}$ Traducción propia.

${ }^{6}$ Traducción propia.

${ }^{7}$ Traducción propia. 
representados como si fueran frutos y flores, a la vez que ofrecen la isla al observador (94). Por tanto, las mujeres tahitianas encarnan la seductora tierra que invita a ser explorada, cartografiada, y penetrada. Es de este modo que a las mujeres se les priva de su derecho a actuar libremente para convertirse en parte de ese inmóvil, pero exquisito decorado como objeto de deseo y placer del hombre. Se trata de la representación de la mujer que comparte con el paisaje el mismo grado de pasividad, quietud, y en definitiva, vulnerabilidad que la propia tierra (94). Según el crítico de arte John Berger (1972), es el espectador el protagonista indiscutible del cuadro. Por el contrario, el papel de la mujer se ve reducido al de una mercancía dispuesta a dar la bienvenida y complacer al dueño del lienzo (Rose 1993: 97). El resultado es la mercantilización del paisaje, del arte y del sexo (97), una forma de "ideología visual" (Cosgrove 1985: 47), o en palabras de la geógrafa Catherine Nash (1996), una "visión opresiva" (151) del paisaje.

\section{Paisaje subversivo: una mirada femenina en la cumbre}

This world is but canvas to our imagination. (El mundo no es sino un lienzo para nuestra imaginación $)^{8}$ Henry David Thoreau (A Week on the Concord and Merrimack Rivers, 1849)

El término "ecofeminismo" fue acuñado por Françoise d'Eaubonne en $L e$ feminisme ou la mort (1974) para señalar la preocupación de algunos grupos feministas franceses por la relación entre superpoblación, devastación de la naturaleza y dominación masculina (Anton Fernández 2015; Flys 2015). Calificadas de "plagas", D’Eaubonne no ofrece más que una solución ante la destrucción de los recursos y la

\footnotetext{
${ }^{8}$ Traducción propia.
} 
superpoblación, es decir, el feminismo o la muerte. Desgraciadamente, sus tesis fueron ridiculizadas al pretender poner en relación dos conceptos; naturaleza y feminismo, que en la época se consideró no guardaban la más mínima relación (Puleo 2013; Antón Fernandez 2017). Al hilo de este interés por explorar en los textos literarios las relaciones humanas con la Naturaleza, uno de los precursores de la ecocrítica, Lawrence Buell (1995) establece cuatro grandes áreas temáticas: (1) el medioambiente no humano no es un mero marco, sino un elemento íntimamente ligado a la historia humana, (2) el interés humano no es el único interés legítimo, (3) la responsabilidad humana por el medioambiente forma parte de la orientación ética del texto, (4) el medioambiente es más un proceso dinámico que un elemento estático (citado en Sanz 2015: 293). En la misma línea, Susan Griffin (2015) defiende que "la existencia humana se encuentra inmersa en la naturaleza, depende de ella y es inseparable" (140). Por otro lado, para la filósofa feminista Alicia H. Puleo (2013), la clave reside en si "nuestra mirada sobre la Naturaleza tiene género" (18). Así es que, de la combinación de literatura, feminismo y ecología, Antón Fernández (2017) presenta cuatro preguntas a formularse ante un texto literario, que serán a su vez las que planteo al texto de Bird: la relación del ser humano con el entorno, la naturaleza animal no humana, el tratamiento de la figura humana, y la representación del cuerpo humano. De su detenido estudio sugiero que la mujer fue abandonando el papel de mero decorado del paisaje, objeto de la mirada masculina, para pasar a ver por ella misma, con sus propios ojos. De esta forma alternativa de contemplar el paisaje presento, además, como elemento más transgresor, que es el cuerpo masculino lo que se identifica con la tierra, en contraposición a la tradicional identificación en Occidente de la Naturaleza con el cuerpo de la mujer.

\footnotetext{
${ }^{9}$ Traducción propia.
} 
3.1 Relación del ser humano con el entorno

La profesora Patricia Vieira (2015), en su conferencia "Sabios en las cumbres: la montaña como exilio de la modernidad", describe la paradoja que presenta "la montaña" con el advenimiento de la industrialización y la pérdida de la conexión del ser humano con la naturaleza. Si bien es cierto que por un lado se identifica a la montaña con lo salvaje y peligroso, puesto que en ella habitan esos seres que no pertenecen a la sociedad moderna y organizada: lobos, dragones, enanos y brujas, también se identifica a esta con lo divino, y a modo de ejemplo, Vieira cita el monte Olimpo; sede de las divinidades griegas, el monte Ararat; elemento clave del folklore armenio, el monte Sinaí; en el que se dice que Moisés recibió los diez Mandamientos. Contrariamente a la crítica que presenta Antón Fernández (2017) acerca de que parece que viviéramos de espaldas a la naturaleza a juzgar por el tratamiento que se hace en la literatura actual de ella: "a lo sumo la concebimos como si fuera un escenario estático, perenne e infinito en el que se enmarcan las peripecias humanas" (58), en el texto de Bird, la montaña se describe efectivamente como refugio contra la monotonía de las ciudades, fuente de salud y bienestar, y como encuentro feliz con una naturaleza en su estado más puro. Como consecuencia de esta sensación de libertad y distanciamiento físico con respecto a las normas y constricciones sociales, Bird da rienda suelta a su mirada.

Consumida buena parte del siglo XIX, y en plena efervescencia de la literatura de viajes, Isabella Bird recoge en La vida de una mujer en las Montañas Rocosas (1879) la colección de cartas que envió a su hermana en un su periplo por el Nuevo Mundo. Habiéndose demostrado que las cartas sufrieron modificaciones desde su redacción hasta su publicación siete años más tarde, se considera improbable o directamente 
imposible buena parte de lo que el lector puede encontrarse en dicha correspondencia (Chubbuck 2002), destacando entre otros, su romance con el desesperado trampero, Jim de las Montañas. No obstante, resulta innegable la solidez de la historia de superación y empoderamiento que Bird presenta con la crónica del ascenso al pico más alto de la cadena montañosa americana.

Narrada en primera persona, de esta novela epistolar se desprende un afecto por la naturaleza difícilmente superable. A ese respecto, Buell (2001) acuña el término "placeconnectedness" (791-936), y plantea cinco formas de vinculación con los lugares. La autora implícita ${ }^{10}$ podría estar adscrita a una de ellas, la cuarta, que denomina "identityshaping places" (880), es decir, aquellos espacios con los que las personas sienten una vinculación especial hasta el punto que marcan un antes y un después en su vida. Lo cierto es que, de niña, Bird se vio obligada a cambiar el verde distrito de Cheshire, en el que nació, por el sofocante y superpoblado ambiente de Birmingham (Barr 2015: 25182519). Incluso podría hablarse de enfermedad ligada al espacio habitado, es decir, en el campo disfruta de buena salud, mientras que en la ciudad cae enferma ${ }^{11}$ (Barr 2015: 2532). En nuestra obra objeto de análisis, con frecuencia no queda claro dónde termina la descripción real del espacio, y dónde comienza la ficticia ${ }^{12}$ : "las montañas, las Sierras tan ensoñadas" (13), y "por fin estoy en un lugar como el que anhelaba pero que excede en todo a lo imaginado" (61). De hecho, hablando del lago Tahoe, lo define como "lugar de ensueño para quedarse mirándolo una vida entera entre suspiros” (9). Esta predilección por la Naturaleza no parece, sin embargo, un elemento nuevo en su

\footnotetext{
${ }^{10}$ Garrido (2007) afirma que se distingue tanto del autor como del narrador, y se define como "la imagen que el autor real proyecta de sí mismo dentro del texto" (115-116).

${ }^{11}$ En relación a la "enigmática" enfermedad de Bird, véase: Checkland 1996; Chubbuck 2002; Barr 2015.

${ }^{12}$ Camarero (2010) y Álvarez Méndez (2010) confieren al espacio "carácter de signo" y lo dotan de tres dimensiones: el espacio del objeto o del referente, es decir, del mundo exterior (Las Rocosas); el espacio del discurso o significante, es decir, el texto propiamente dicho, y el espacio de la historia, es decir, del significado, producto de la imaginación (Las Rocosas descritas y narradas por la autora implícita).
} 
existencia. A este respecto, Marie Dronsart, autora de Des Grandes Voyageuses (1894) califica de "viril" (citado en Rogers y Thébaud 2008: 9) la educación que recibían las mujeres inglesas, con la práctica de la marcha y la equitación, lo que las predisponía a viajar y recorrer el mundo sin temor. Ilustrativo de esta "virilidad", es la confesión de la propia jinete acerca de que las Rocosas constituyen la "perfección misma: están presentes todos los requisitos para la salud, incluyendo muchos caballos y terrenos herbosos para cabalgar" (62). En el caso de Bird, se da la casualidad de que recibió una educación especialmente "masculina". Olive Checkland (1996) relata que el reverendo Edward Bird, en el desempeño de su anterior oficio como abogado en India, perdió a su mujer y primer vástago. De vuelta en Inglaterra, siendo Isabella Bird la hija mayor del segundo matrimonio del reverendo, ésta pudo disfrutar de las oportunidades reservadas a ese hipotético primogénito varón fallecido. Decidida a emularle en todo, Bird se convirtió de este modo en su más leal acólita, tanto como compañera de paseos a caballo, como de viajes al extranjero (Checkland 1996: 3).

Según avanza la narración, así también lo hace la expresión de este sentimiento profundo de amor por la Naturaleza que va en crescendo a medida que la protagonista se va aproximando físicamente a las Rocosas. Prueba de su aflicción ante la feroz batalla lidiada por la Naturaleza contra la civilización es la carga negativa que se desprende de la colección de adjetivos que emplea para referirse al impacto de la furia del oro: "agua fangosa [...] el aun más fangoso Sacramento" (10). Para la autora, esta "civilización" genera "profundas cicatrices" (9) en la tierra, y supone una verdadera amenaza para el medio y los que en ella habitan. Con todo, el alegato en favor de la tradicional agricultura que "restaura y embellece" (147), frente a la moderna minería que "destruye y devasta" (147) constituye una de las más rotundas y valientes declaraciones en defensa del medio. Según la autora, ésta no solo vacía y afea la tierra, 
sino que además "marchita el corazón y alma de los hombres" (147). Por otro lado, la destrucción, sin embargo, no contenta con aniquilar la fauna y la flora autóctonas, crea tensión entre sus habitantes, es decir, entre la población indígena y los ávidos colonos. Posteriormente, la viajera pasa a describir las desventuras de los indios Digger a los que define como "incongruencia en medio de los signos de una omnipotente civilización" (11), y en peligro de extinción "ante el empuje de la raza blanca" (11). Ante tal escalda de "modernidad", ni los primeros pobladores de California parecen sentirse a gusto, de hecho, la aventurera recoge el testimonio de uno de ellos que afirma alejarse "de los lugares a que llegaba a medida que se volvían demasiado civilizados" (13). En cuanto a la corrupción del "alma" a la que aludía en líneas previas, la autora de las misivas señala como máximo responsable, junto a la fiebre del oro, al whisky, "de todo el mal, la violencia y de la mayoría de tiroteos (140). Íntimamente relacionado con ese caldo alcohólico, la exploradora victoriana afirma sentirse impresionada por el número de "salones" de Colorado y la abundancia de "los característicos individuos ociosos de toda ciudad fronteriza" (108), caldo de cultivo de muertes inútiles: "acuchillamientos, disparos, duelos de pistolas, que acaecían casi a cada hora en sus cuchitriles de venta de bebidas" (27). En contraposición, la naturaleza más remota e inexpugnable de las montañas en las que nunca se ha escuchado un hacha de leñador, constituyen para la viajera un remanso de paz, tranquilidad, armonía con el medio y consigo misma, que la colman de salud y bienestar. Define el aire de Wyoming y de Boulder como "el elixir de la vida (19), y muy "salutífero" (61), cuya vida "embriaga" (83). Difícil no sentirse exultante viviendo al aire libre, montada a lomos de su yegua fiel, ataviada con su cómodo traje hawaiano, y durmiendo, si el tiempo lo permite, "bajo las estrellas sobre un lecho de ramas de pino" (83), "sin relación alguna con las Midlands" (85). 
A grandes rasgos, Bird presenta a una heroína en armonía con el medio, cuya historia se encuentra íntimamente ligada a la historia del ecosistema del que forma parte, aunque siempre alejada de esa idea romántica de paisaje domesticado, mero decorado. De hecho, Bird aboga por una naturaleza en estado puro, salvaje, no contaminada por la civilización. En definitiva, en el texto de Bird, el medio natural es dotado de realidad propia, como si de un personaje más de la obra se tratara. De ahí que su trabajo pueda en última instancia interpretarse como una llamada de atención al estado de riesgo del entorno natural y al papel humano en su degradación.

3.2 Naturaleza animal no humana

Por un lado, el rechazo y la denuncia de la violencia contra los animales es una constante en la obra de Bird, y por el otro, sus reiteradas muestras de solidaridad tampoco pasan desapercibidas. A ese respecto, es fundamental el apunte del filósofo Peter Singer acerca de lo acertado de considerar la capacidad de sentir dolor, y no la capacidad de razonar, la clave que guía el comportamiento moral en el trato con otros seres vivos (citado en Sanz 2015: 302). Así que la autora se muestra muy consciente de las desastrosas consecuencias de la matanza del búfalo por parte del hombre blanco. No solo como pérdida de la biodiversidad, sino como desencadenante de un conflicto de intereses que desemboca en una guerra abierta entre aquellos y los indios, puesto que "el búfalo es su principal medio de sustento" (33). A su vez, el trato al que los humanos en general someten al ganado le genera aflicción calificando el sistema de "casi terrorista" (99). Muestra del profundo cariño que profesa por la ganadería ecuestre, la autora bautiza a su más querido y leal poni indio bayo con el curioso nombre de 
"Berdie", en clara referencia a su propio apellido, y como si de una hija se tratara, la describe como "una pequeña belleza, con piernas de hierro, resistente, amable y lista" (104). La viajera confiesa que lo suyo ha sido amor a primera vista puesto que "solo llevábamos dos días juntos y éramos firmes amigos, entendiéndonos por completo" (63). De Ring, el devoto perro de Mountain Jim, la amazona afirma que "posee un noble rostro de expresión humana" (73). Así que Jim se dirige a él como si de un humano se tratara. Por lo demás, es cariñoso y busca el contacto y la caricia de sus guardianes. Por otro lado, la proximidad y el contacto con la naturaleza en su estado más puro tampoco parece asustar a la aventurera, más bien todo lo contrario. De hecho, narra la presencia frente a ella "de un alce con cuernos con ramificaciones de tres pies de longitud" (47) sin inmutarse, y descubre que la noche pasada los osos habían arrancado arbustos de cerezas a unas pocas yardas de su tienda. A ese respecto, sus muestras de empatía hacia el mundo animal no humano son constantes: en una ocasión "como el caballo estaba nervioso y parecía como temeroso de ese camino, salí del mismo con la idea de un breve descanso" (16). En otra ocasión, al ver pasar a una osa con sus retoños, acalla y calma a su caballo, para evitar asustarlos. A su vez, a los perros de la pradera les confiere características humanas: "estas criaturas obran como vigilantes mientras toman el sol" (29), incluso "hablan": "daban un grito de advertencia a la vez que sacudían la cola y con un elegante gesto de sus patas posteriores penetraban a toda prisa en el orificio" (30). Cada párrafo del texto rezuma un profundo respeto y admiración por todas las especies, las conoce y se refiere a ellas con una mezcla de ternura poética y precisión científica:

Los arrendajos volaban entre los pinos, cientos de ardillas correteaban por el bosque, las libélulas brillaban cual luminarias vivientes, exquisitas ardillas listadas corrían por los senderos, aunque era algún ocasional altramuz azul cubierto de 
polvo lo que me hacía realmente tomar conciencia de la belleza que puede engendrar la naturaleza. (2014: 17)

Al margen de las recurrentes referencias a la abundante, variada y salvaje fauna en el medio que la rodea, la viajera selecciona a dos animales como compañeros silenciosos a lo largo de todo el texto. Por un lado, destaca el protagonismo de Ring como personaje mascota, compañero habitual de seres solitarios como Jim Mountain. Por el otro, Birdie, el poni indio bayo compañero infatigable de la protagonista. Podría añadirse que con su presencia o su comportamiento complementan o enfatizan las características de ambos héroes. Bird muestra además una preocupación ética por el sufrimiento de los animales: los caballos, el ganado, incluso los osos, y efectivamente, todas las especies animales son tratadas como seres vivos y "sintientes", y comparten la capacidad y necesidad humana de cuidar y ser cuidados (Antón Fernández 2017: 55), lo que evoca la ética del cuidado ecofeminista y su propuesta de relación dialógica con todos los seres (humanos o no) que comparten este planeta (Flys 2013: 93).

\subsection{Tratamiento de la figura humana}

Partiendo del aserto fundamental de que la dominación de las mujeres y la dominación de la naturaleza responden al patrón de comportamiento del patriarcado en el que el hombre se identifica con la razón, mientras que la mujer se halla indisociablemente unida a la naturaleza, la novedad del ecofeminismo se encuentra para la autora de Eva en el Laberinto: una reflexión sobre el cuerpo femenino (2012), Olaya Fernández Guerrero, en que aporta una perspectiva de género que revaloriza la 
experiencia femenina con respecto al entorno natural (243). Es precisamente en este apartado en el que cobra todo su sentido el estudio del paisaje observado por una mujer (blanca y heterosexual). De hecho, contrariamente al binomio Naturaleza/Mujer, la obra elegida constituye un magnífico ejemplo de la mujer no tanto como parte del entorno paisajístico, o de la analogía Madre/Tierra, sino como observadora, artista, e intérprete de un lenguaje que hasta entonces había sido mayormente descrito y dominado por hombres, de ahí su trascendencia. Fernández Guerreo (2012) añade que gracias a la simbiosis feminismo/ecología, la lucha feminista se ve complementada con la lucha ecológica contra la dominación del entorno, permitiendo una comprensión más en profundidad de los mecanismos de dominación. A su vez, y en la misma línea que Fernández Guerreo (2012), Ma Antonia Bel Bravo en su artículo; "Ecofeminismo: una nueva manera de mirar la naturaleza" (2016), subraya la recurrencia a lo largo de la historia de la visión dualista del mundo: Naturaleza/Razón, Cuerpo/Mente entre otros. Lo cierto es que, llevada esa visión al plano del conservacionismo ambiental, Bel (2016) denuncia que se presente la naturaleza como principio contrario al ser humano. De ahí, su identificación con el enemigo cuya conquista resulta ineludible para hacerse con el botín, es decir, sus recursos. En esa atmósfera, Bel (2016) señala que la clave de la actuación histórica de las mujeres con respecto al dualismo Naturaleza/Cultura descansa en el hecho de que las actividades tradicionales de las mujeres: maternidad, cocinar, cultivar, curar, etc. son tanto sociales, es decir culturales, como naturales, por tanto, la tarea de un feminismo ecológico debería ser a su juicio la de configurar una teoría y una praxis antidualista.

En relación al tratamiento de la figura humana que se desprende de la obra seleccionada, Antón Fernández (2017) se pregunta cómo se representan los cuerpos, si se trata de cuerpos finitos, con necesidades, y si además es posible detectar diferencias 
de género, e identificar estereotipos (55). En este apartado, cobra especial relevancia la cuestión acerca de si el texto recrea la dicotomía Naturaleza/Cultura. A su vez, Antón Fernández (2015) señala en su tesis el origen de la dualidad Cuerpo/Mente. Para ello recurre a la interpretación mítica presentada en términos de género por Fernández Guerrero (2012: 29-66) quien hace uso de la mitología órfica, según la cual, en la batalla que enfrentó a los Titanes contra Zeus para hacerse con el control del Olimpo, a pesar de la victoria de éste, aquellos capturaron y devoraron a uno de los hijos predilectos de Zeus, Dioniso. Como venganza, Zeus eliminó a los Titanes, y sobre sus cenizas creó al género humano. Así es como Fernández Guerrero (2012) explica el dualismo en la concepción del ser humano, mientras que el cuerpo es heredero de la parte impura de los Titanes, el alma, de naturaleza divina, corresponde al legado de Zeus (29). Una vez ideada la dicotomía Cuerpo/Mente, el dualismo Varón/Mujer como sinónimo de Cultura/Naturaleza, señala Antón Fernández (2015), también se encuentra presente en nuestra cultura occidental desde la Antigüedad. En esta división dicotómica, a la mujer le ha correspondido, señala Puleo (2013), ser la expresión de la Naturaleza, puesto que su constitución biológica se encuentra dominada por procesos metabólicos que no requieren actividad consciente. Por el contrario, al varón se le asocia con la Razón y la Cultura, cuyo valor es muy superior al de la Naturaleza, razón por la que se legitima su dominación y, por ende, la persistencia de la desigualdad. De acuerdo a la filósofa francesa Geneviève Fraise (2002), sin embargo, "es por el cuerpo y no por la razón como los hombres y las mujeres se distinguen fundamentalmente" (212), y siguiendo este principio, Bird presenta dos ejemplos masculinos: Jim de las Montañas y Thomas Chalmers. Este último ilustra a la perfección la máxima anterior. La viajera lo describe poco agraciado. Lo califica de "levemente inteligente, muy obstinado" (42). En cuanto a estereotipos sexuales, el elemento más transgresor resulta la ineptitud de 
Chalmers como colono en el Oeste tras casi una década de experiencia, en contraposición a la superioridad en seguridad, rigor y experiencia de la recién llegada protagonista femenina. La viajera le acusa de carecer de habilidad alguna para orientarse en su labor de guía hacia Estes Park, de ahí que sea ella quien deba indicarle que el camino escogido no es el correcto. Lo más irritante resulta para la aventurera que la infravalore, "enciende el fuego porque me cree tan estúpida como para no saber hacerlo" (38). En cuanto a Jim, el desesperado trampero, encarna la exquisita combinación de inteligencia y sensibilidad echada a perder por unas condiciones de vida extremas y el abuso del alcohol, una especie de reverso de la figura masculina anterior. La viajera lo describe como "un hombre ancho y sólido, de talla mediana" (65). La aventurera sentencia que "su rostro era notable" (65). En cuanto a su comportamiento, Jim es educado y adopta la actitud de un "galante caballero" (66). Por otro lado, cuenta con una habilidad innata para desenvolverse en ese espacio que la autora tanto venera: "es un verdadero "hijo de la naturaleza" (173). Es un hombre sensible y sincero, especialmente hablando a propósito de su juventud y de la tragedia que le había conducido a esa vida de delincuencia. Ama intensamente la naturaleza y a los niños, tiene humor, emite notables juicios agudos, e incluso escribe poesía. Difícil imaginar una representación masculina más acertada, sin embargo, "es demasiado tarde. Ya no puedo cambiar" (159), sentencia Jim. A grandes rasgos, el discurso de Bird se encuentra salpicado de apreciaciones y diálogos de un tinte paternalista importante. Son reiterados los comentarios sobre "el hábito de respetuosa cortesía hacia las mujeres" (20) incluso en los lugares más apartados de la civilización en los que la misma Bird excusaría exabruptos o comportamientos indecorosos. En opinión de la geógrafa norteamericana Karen M. Morin (2007), el texto de Bird se hace eco de los comportamientos que recuerdan la "ubicuidad" imperialista para subrayar la noción de que la "metrópoli" 
gobierna incluso en los lugares más remotos. Por otro lado, se alaba la figura del hombre galán, cortés, y especialmente sensible, pero a su vez, éste debe ser consciente del cambio en el status quo de la mujer, cada vez más interesada en demostrar al mundo su valía. Es precisamente en la línea del aumento del protagonismo femenino en la esfera pública que Puleo (2013) presenta una interpretación alternativa a la del mito del Minotauro, puesto que Ariadna se ha convertido en la verdadera protagonista de la historia (7-8). Esta "nueva Ariadna" no desea conformarse con un papel secundario en ayuda de Teseo, sino que ella también se adentra en el laberinto. De hecho, Puleo (2013) reivindica que Ariadna está siendo la transformadora de la cultura en la era del cambio climático. Como consecuencia, a Bird la Naturaleza no le produce pavor, sino simpatía. Por otro lado, contrariamente a lo que cabría esperar de una lady victoriana, la viajera no evita el trabajo, ni siquiera el manual. De hecho, ante la costumbre generalizada en el Oeste de trabajar independientemente del género, se siente aliviada de no tener que escuchar frases hechas del tipo: “¡Oh, no debería hacer usted eso!" (171) o “ $\mathrm{O}$, permítame que yo haga eso! (171). Igualmente, aunque ella misma menciona que se dividen el trabajo de cocina, hay ciertas tareas que "ellos" siguen considerando inadecuadas para una mujer ahondando en la idea anteriormente citada sobre la omnipresencia colonialista:

Los hombres no quieren que haga nada que a ellos les parezca arduo o inadecuado, tal como ensillar un caballo o traer agua [...] te divertiría entrar en nuestra pequeña y desmañada cocina poco antes del desayuno de ayer. Nos hubieses visto a Mr. Kaven ocupado en freír el venado, a mí lavando los platos de la cena y Mr. Buchan secándolos, o bien a ambos jóvenes ocupados con el fuego mientras yo friego el suelo. (2014: 166)

Por otro lado, un elemento que merece especial atención por la relevancia que adquiere en el texto es la indumentaria femenina, importante herramienta de control 
social de la mujer. Claire A Roche (2015), en su sobresaliente tesis sobre las mujeres en sus travesías alpinas, narra desde una perspectiva histórica la compleja coyuntura a la que se enfrentaban aquellas al verse abocadas a sacrificar cierto grado de "feminidad" al tiempo que realizaban actividades consideradas poco o nada "femeninas" debido, en gran medida, al extraordinario esfuerzo físico que requieren (24). A Roche apunta que el código de vestimenta venía determinado por el tipo de compañía de la que disfrutaban en cada momento, debían conservar un aspecto femenino acorde con los criterios de feminidad europeos, pero en la montaña prevalecía el pragmatismo (25). Valga como ejemplo el ágil cambio de atuendo de la viajera al aproximarse a un emplazamiento habitado: "desmonté, me puse una falda larga y volví a montar sentada de lado, aunque la colonia no parecía un lugar en que fuera necesario manifestar deferencia a los prejuicios" (117). Nótese igualmente el detalle de la modificación en la forma de montar. Primero a horcajadas, más tarde a la manera socialmente considerada femenina, es decir, de lado. Lo cierto es que en una época en la que el aspecto de la mujer constituía uno de los pilares de su feminidad (A Roche 2015: 242), Bird, considerada una de las primeras mujeres occidentales en enfundarse unos "pantalones turcos" (Chubbuck 2002: 180-181), se detiene, desde las primeras páginas, en detallar en qué consiste su particular "atavío hawaiano": "chaqueta corta, falda atada a la cintura y pantalones turcos" (14) para ahuyentar críticas veladas a su aspecto. Imprescindible igualmente el pañuelo atado a la cara y la sombrilla a mano, para evitar el bronceado de la piel considerado igualmente poco femenino (A Roche 2015: 242): "vestía mi ropa hawaiana de montar con un pañuelo atado sobre mi cara y el protector de mi sombrilla plegado y atado sobre mi sombrero" (49-50). Otro elemento a cuya descripción la autora consagra tiempo y espacio es el alojamiento y menaje. Son abundantes las referencias al estado y características de los espacios cerrados y aposentos, en su mayoría de una 
calidad muy cuestionable: "El techo tenía agujeros, los maderos estaban sueltos, iy un extremo del recinto se hallaba en parte abierto!" (37-38). Si bien es cierto que los escritores, independientemente de su género, consagran sus textos, según A Roche (2015), a temáticas que les son familiares, en los relatos de mujeres abunda particularmente, la temática doméstica: estancias, alimentación e indumentaria, cuestión que Bird describe con suma elocuencia en el siguiente pasaje:

El buey que me dieron era duro y grasiento, la mantequilla era puro aceite, y el buey y la mantequilla estaban negros de tanta mosca viva o medio ahogada. El grasiento mantel también estaba negro de moscas y no era de extrañar que los huéspedes tuviesen aspecto melancólico y anhelasen escapar de allí [...] la idea de un hotel, sabiendo cómo eran en América, fue formidable dado el presente estado de mi vestuario. Decidí, pues, traer mi saco, así como mis maletas, para que no se me rechazara debido a mi desastrada ropa. (2014: 35)

En esa misma línea, la profesora Mary Louise Pratt (1992), señala que, contrariamente al ansia de conquista masculina que cede poco o ningún espacio a la domesticidad, la reivindicación femenina consiste en "la consecución de un espacio público, personal, un imperio del tamaño de una habitación”"13 (159-160) puesto que, según Morin (2008), recrear el mismo ambiente de sus propias casas les permite sentirse a gusto allí donde se desplazan (70). De hecho, mientras el objetivo de un buen número de hombres consiste en "coleccionar y poseer" lo que encuentran a su paso, Pratt (1992) señala que las mujeres que viajan buscan ante todo "recuperarse" en clara referencia a la huida de una situación personal anterior compleja (159-160) derivada del exceso de celo y control familiar y/o social.

Por tanto, el discurso de Bird oscila, grosso modo, entre el intento desesperado de ruptura con la tradición patriarcal valiéndose del peculiar emplazamiento (Las Rocosas

\footnotetext{
${ }^{13}$ Traducción propia.
} 
en el caso de Bird) y la irremediable sumisión a los más clásicos mecanismos de control social femeninos. Con respecto a la postura ambigua de la viajera en lo concerniente al decoro al protagonizar ciertas escenas transgresoras, Morin (2003) sostiene que el texto se mueve entre el respeto al estricto código de conducta victoriano y el relato heroico de aventuras en el que la viajera encarna al héroe sin renunciar a su "feminidad" (212). Las críticas literarias feministas Sandra M. Gilbert y Susana Gubert (2000) tratando precisamente de proporcionar una explicación a esta llamativa oscilación, afirman que es consecuencia del intento de las mujeres por "encajar" en un mundo dominado por hombres. El resultado es a su juicio "anómalo, inexplicablemente aislado y peculiarmente raro"14 (48).

\subsection{Representación del cuerpo humano}

La fotógrafa y escritora surrealista Claude Cahun en clara referencia a una reinterpretación de la máxima cartesiana: "me veo, luego existo" (citado en Alario 2008: 110), alude a la importancia que para la autoconstrucción de la identidad tiene la mirada, especialmente para la mujer, a quien se le ha negado tradicionalmente el control de ésta. En la misma línea, para $\mathrm{M}^{\mathrm{a}}$ Teresa Alario (2008), estudiosa de la figura de la mujer en el Arte, la conciencia de verse a sí misma implica también comenzar a ver el mundo y narrarlo de otro modo (115-116). En una época la que el mero hecho de hablar con desconocidos del otro género contravenía las convenciones sociales, en el texto se suceden las escenas de contacto físico. Especialmente llamativas son las descripciones del ascenso a Long's Peak donde la autora confiesa que, unas veces, Jim la "montaba

\footnotetext{
${ }^{14}$ Traducción propia.
} 
sobre sus hombros" (79) para facilitarle la subida, en otras ocasiones, se "hallaba atada a Jim" (76) para evitar extraviarse. En uno de los momentos más críticos del ascenso Jim incluso "tiró de [ella] como de un fardo, con la fuerza bruta de sus músculos" (76). Además, la narración se encuentra salpicada de referencias veladas a las formas un tanto atrevidas de Jim, dejando entrever el peligro claramente sexual que corre la protagonista. Peligro que, sin embargo, lejos de retraerla, provoca atracción magnética: "Jim me hizo volver al camino y el encuentro que había empezado con un tiro de pistola terminó de modo muy placentero. Fue una cabalgada casi irreal, para no ser olvidada, aunque no hubo peligro" (177). Probablemente se trata esta de la estrategia literaria que Bird ha escogido para allanar el camino hacia lo que podría considerarse la escena culminante de su heroico peregrinaje, que no es otra que una experiencia sexual, como así se infiere del retrato de la pareja alrededor de una hoguera, en un lugar remoto, bajo las estrellas mientras Jim fuma. De hecho, en la misma carta en la que describe el momento épico de la coronación de Long's Peak (Chubbuck 2002), la autora afirma que “es una de las más notables montañas, pero en la imaginación crece hasta ser más que una montaña" (69) en clara referencia al órgano genital masculino (DeVine 2007). La viajera reitera que "de entre ese vasto mar de montañas" (49), es Long's Peak "único en grandeza imperial" (49). Resulta en ese sentido tan curioso como novedoso comprobar el protagonismo que alcanza la genitalidad masculina en el texto, no solo porque la literatura ha sido más proclive a mostrar la femenina, sino porque la virilidad de Jim, el personaje masculino por excelencia de la obra, ha sido reducida a una simple forma fálica. Lo cierto es que el relato de Bird echa por tierra la tradicional representación de la mujer como un elemento pasivo y estático más del paisaje, concebido para proporcionar placer al espectador masculino, puesto que en el texto se representa a la mujer como un ser que busca placer a través de la experimentación de la sexualidad, lo 
que la conduce a un empoderamiento sexual. En esa misma línea, Bird proclama a la viajera "novia de la naturaleza" (Carretero 2005: 182) lo cual contribuye a cuestionar la percepción de esta como madre cariñosa y protectora para convertirla en unas atractivas “curvas” masculinas. Además, sugiero que, de la combinación del cuarteto: naturaleza, género, literatura y ecología, Bird presenta una visión tan subversiva como moderna del paisaje. En primer lugar, la Naturaleza que describe no se reduce a un simple escenario como en el caso del paisaje de los grandes exploradores masculinos, a descubrir, penetrar y dominar, sino que se trata de un componente imprescindible de nuestra existencia a respetar y valorar como a un igual. A su vez, Bird reivindica la igualdad y la autonomía de las mujeres al auto conferirse el derecho a ser protagonista. En cuanto al paisaje, Bird lo contempla desde una posición si no femenina, al menos alternativa a la tradición occidental, planteando una metáfora de la anatomía genital masculina con un determinado lugar geográfico. Finalmente, la aportación de Bird contribuye a reconciliar la dicotomía Naturaleza/Cultura, puesto que, manifiesta su más sincero respeto y admiración por la Naturaleza al mismo tiempo que hace alarde de un conocimiento profundo de ella, reivindicando así su doble pertenencia como mujer a la Naturaleza y a la Cultura.

\section{Referencias bibliográficas}

Alario Trigueros, $\mathrm{M}^{\mathrm{a}}$ Teresa. 2008. Arte y feminismo. Donostia: Nerea. Kindle.

Álvarez Méndez, Natalia. 2002. Espacios Narrativos. León: Universidad de León. 2010. "Hacia una teoría del signo espacial en la ficción narrativa contemporánea". Biblioteca virtual universal: 550-570. http://www.biblioteca.org.ar/libros/154801.pdf [03/12/2018].

Antón Fernández, Eva. 2015. ¿Cambio de roles de género en el cambio de siglo? Un análisis comparativo de las narrativas francesa y española (1990-2010). Tesis. 
Universidad

de

Burgos.

http://riubu.ubu.es/bitstream/10259/4627/1/Antón_Fernández\%20.pdf [5 enero 2019]

. 2017. "Claves ecofeministas para el análisis literario". Revista de investigación y divulgación sobre los estudios de género 21: 45-74. http://bvirtual.ucol.mx/descargables/803_ilovepdf_com-47-76.pdf [10/10/2018].

Barr, Pat. 2015. A Curious Life for a Lady: The Story of Isabella Bird. London: Faber \& Faber. Kindle

Bel Bravo, M.A. 2016. "Ecofeminismo: una nueva manera de mirar la naturaleza". Arbor, 192 (778): a304. doi: http://dx.doi.org/10.3989/arbor.2016.778n2007 [03/03/2019].

Berger, John. 1972. Ways of Seeing. London: Penguin.

Bird, Isabella L. 2014. La vida de una mujer en las Montañas Rocosas. Carlos Ezquerra (trad.). Barcelona: Erasmus clásicos en el presente.

Buell, Lawrence. 1995. The Environmental Imagination. Cambridge: Harvard University Press.

2001. Writing for an Endangered World. Literature, Culture and Environment in the US and Beyond. Massachusetts: Harvard University Press. Kindle

Cahum, Claude. 2002. "Confidences au miroir". François Leperlier (ed.). Des écrits: 161-436.

Camarero, Jesús. 2010. "Escritura, espacio, arquitectura: una tipología del espacio literario". Biblioteca virtual universal: http://www.biblioteca.org.ar/libros/154572.pdf [09/01/2019].

Carretero González, Margarita. 2005. "I Am Nature's Bride: Orlando and The Marriage of Nature and Culture". In-Between: Essays and Studies in Literary Criticism 14: 259-268.

2010. "Ecofeminismo y análisis literario". Ecocríticas: Literatura y Medio Ambiente: 177-189.

Checkland, Olive. 1996. Isabella Bird and a Woman's Right: To Do What She Can Do Well. Aberdeen: Scottish Cultural Press.

Chubbuck, Key. 2002. Letters to Henrietta. Isabella Bird. London: John Murray.

Cosgrove, Denis E. 1983. "Towards a Radical Cultural Geography: Problems of Theory". Antipode 15: 1-11. 
1985. "Prospect, Perspective and the Evolution of the Landscape Idea". Transactions of the Institute of British Geographers: New Series 10: 1-45.

DeVine, Christine. 2007. "Isabella Bird and Mountain Jim: Geography and Gender Boundaries in A Lady's Life in the Rocky Mountains". Nineteenth-century gender studies. https://www.ncgsjournal.com/issue32/devine.htm. [30/01/2019].

Dronsart, Marie. 2019. Des Grandes Voyageuses. Wentworth Press. Kindle.

Fernández Guerrero, Olaya. 2012. Eva en el laberinto. Una reflexión sobre el cuerpo femenino. Málaga: Servicio de Publicaciones de la Universidad de Málaga.

Flys Junquera, Carmen. 2013. “"Las piedras me empezaron a hablar”: una aplicación literaria de la filosofía ecofeminista". Feminismo/s: 89-112.

2015. "Ecocrítica y ecofeminismo: diálogo entre la filosofía y la crítica literaria". Ecología y género en diálogo interdisciplinar: 307-320.

Fraise, Geneviève. 2015. La controversia de los sexos. Identidad, diferencia, igualdad y libertad. Madrid: Minerva. Kindle

Garrido Domínguez, Antonio. 2007. El texto narrativo. Madrid: Síntesis

Gilbert, Sandra. M. y Susana Gubar. 2000. The Madwoman in the Attic: The Woman Writer and the Nineteenth Century Literary Imagination. 2nd ed. New Haven: Yale University Press. Kindle

Griffin, Susan. 2015. Women and Nature. The Roaring inside Her. New York: Open Road Media. Kindle

Macfarlane, Robert. 2017. Mountains of the Mind. The History of a Fascination. London: Granta Books. Kindle

Morin, Karen M. 2003. "Narrating Imperial Adventure. Isabella Bird's Travels in the Nineteenth Century American West". Western Places, American Myths: How We Think About the West: 204-222.

2008. Frontiers of Femininity. A New Historical Geography of the Nineteenth-Century American West. New York: Syracuse University Press.

Nash, Catherine. 1996. "Reclaiming Vision: Looking at Landscape and the Body". Gender, Place and Culture 3.2: 149-169.

Pratt, Mary Louise. 1992. Imperial Eyes: Travel Writing and Transculturation. London: Routledge.

Puleo, Alicia H. 2013. Ecofeminismo para otro mundo posible. Universidad de Valencia: Cátedra. Kindle 
Roche, Clare A. 2015. The Ascent of Women: How Female Mountaineers Explored the Alps 1850-1900. Tesis. University of London. https://core.ac.uk/download/pdf/33269169.pdf [25/01/2019].

Rogers, Rebecca y Françoise Thébaud. 2008. "Voyageuses", Clio 28: 5-16. https://journals.openedition.org/clio/7563 [10/11/2018]

Rose, Gillian. 1993. Feminism and Geography: the Limits of Geographical Knowledge. Cambridge: Polity Press. Kindle

Sanz, Teófilo. 2015. "La Ecocrítica, vanguardia de la crítica literaria. Una aproximación a través de la ecoética de Marguerite Yourcenar". Ecología y género en diálogo interdisciplinar: 291-305.

Singer, Peter. 1995. Ética práctica. Cambridge: Cambridge University Press.

Vieira, Patricia. 2015. "Sabios en las cumbres: la montaña como exilio de la modernidad". IV jornadas sobre literatura, cine y montaña. https://ehutb.ehu.eus/video/58c670dff82b2b79178b45a0 [04/11/2019]. 